\title{
FUNCTIONAL RESULTS AFTER VOCAL CORD MEDIALIZATION PROCEDURE WITH SCULPTURED SILICON BLOCK
}

\section{KC Toran, Shrestha S}

\section{ABSTRACT}

Medialization technique has remained as a mainstay for the treatment of glottal insufficiency. This form of laryngeal framework surgery not only improves the quality of voice but also protects the lungs from aspiration.

We present six patients who underwent vocal cord medialization surgery. Of the six patients only one patient had required revision surgery. Since these form of surgeries are performed under local anesthesia the quality of the voice can be assessed per-operatively. This kind of surgery appears to be still new in the Nepalese context.

\section{Key Words: Isshiki Thyroplasty, Laryngoplasty, Laryngeal framework surgery, vocal cord paralysis, Voice disorders, glottal insufficiency.}

\section{INTRODUCTION}

Vocal cord paralysis may be unilateral or bilateral, abductor or adductor. The effect of vocal cord paralysis is dual. If the laryngeal sphincter is incompetent then phonation will be poor and aspiration will be possible. Furthermore, loss of sensation, either in the upper or lower laryngeal compartment will result in aspiration being undetected and aspiration pneumonia will result.

Payr described a method for medializing a paralyzed vocal cord with external access as early as 1915. This method gained worldwide popularity during the 1970s. Later Isshiki modified this technique with a silicon block. ${ }^{1}$ He also coined the term "thyroplasty" type I for this operation, while other authors prefer the name "medialization laryngoplasty".

Materials like fat, liquid paraffin, hydrogen gel, teflon paste,
GAX collagen, hydroxyapetite are being utilized to medialize the paralyzed cord through the endolaryngeal approach. ${ }^{2}$

External medialization materials such as silicon blocks and more recently prefabricated titanium vocal fold implants and Montgomery implants are also in use. ${ }^{3}$ Silicon has remained as an ideal biocompatible material which is in use for medialization surgery over three decades. ${ }^{4}$

The medialization vocal fold surgery is feasible in all the cases of unilateral vocal fold paralysis despite of the different etiology and can be performed under local anesthesia without any discomfort to the patient.

Thyroplasty is an effective procedure for correcting incomplete glottic closure and when performed carefully with proper preoperative assessment then the major complications are virtually nil. ${ }^{5}$

* Kathmandu Medical College Teaching Hospital, Sinamangal, Kathmandu, Nepal.

Address for correspondence : $\quad$ Dr. Toran $\mathrm{KC}$

Dept. of ENT-HNS

Kathmandu Medical College Teaching Hospital

Sinamangal, Kathmandu, Nepal

Email: kc_toran@hotmail.com 


\section{MATERIAL AND METHOD}

Six patients were operated nearly over two year's period between February 2001 and December 2002 at Kathmandu Medical College Teaching Hospital. The following patients were included in the study irrespective of age and sex.

1. Patient who presented with dysphonia due to idiopathic vocal cord paralysis.

2. Iatrogenic vocal cord palsy following head and neck surgeries.

3. Irradiated laryngeal cancer in which no recurrence or residual disease is present.

4. Vocal cord palsy of any cause when aspiration is a problem.

All the patients underwent complete physical examination including laryngeal examination and laryngeal compression tests. In the older age group $\mathrm{x}$-ray soft tissue neck lateral view was carried out to determine the extent of thyroid cartilage ossification. First two patients were the cases of idiopathic cause. The first patient underwent $\mathrm{x}$-ray examination of the chest, soft tissue neck and base of the skull. Panendoscopy was performed under general anesthesia to rule out any obvious pathology. But no blind biopsies were taken. The second patient was a lady of 28 years old in whom x-ray of the chest, soft tissue neck and base of the skull was carried out. In all other patients x-ray chest and $\mathrm{x}$-ray soft tissue neck were done.

None of our patients were advised for CT scan or magnetic resonance imaging.

Pre-operative voice evaluation was performed in all the patients. The subjective voice acquisition parameter scale was made and different scores were given in terms of loudness intensity, pitch and quality of voice (Table I). The principal and co-authors and the patients attendant as a layman did the subjective scoring assessment. 